\title{
Advanced mirror technology development (AMTD) project: 3.0 year status
}

\author{
H. Philip Stahl
}

\begin{abstract}
Advanced Mirror Technology Development (AMTD) is a funded NASA Strategic Astrophysics Technology project. Begun in 2011, we are in Phase 2 of a multi-year effort. Our objective is to mature towards TRL6 critical technologies needed to produce 4-m or larger flight-qualified UVOIR mirrors by 2018 so that a viable astronomy mission can be considered by the 2020 Decadal Review. The developed technology must enable missions capable of both general astrophysics and ultra-high contrast observations of exoplanets. Just as JWST's architecture was driven by launch vehicle, a future UVOIR mission's architecture (monolithic, segmented or interferometric) will depend on capacities of future launch vehicles (and budget). Since we cannot predict the future, we must prepare for all potential futures. Therefore, we are pursuing multiple technology paths. AMTD uses a science-driven systems engineering approach. We mature technologies required to enable the highest priority science AND result in a high-performance low-cost low-risk system. One of our key accomplishments is that we have derived engineering specifications for advanced normal-incidence monolithic and segmented mirror systems needed to enable both general astrophysics and ultra-high contrast observations of exoplanets missions as a function of potential launch vehicle and its inherent mass and volume constraints. Another key accomplishment is that we have matured our technology by building and testing hardware. To demonstrate stacked core technology, we built a $400 \mathrm{~mm}$ thick mirror. Currently, to demonstrate lateral scalability, we are manufacturing a 1.5 meter mirror. To assist in architecture trade studies, the Engineering team develops Structural, Thermal and Optical Performance (STOP) models of candidate mirror assembly systems including substrates, structures, and mechanisms. These models are validated by test of full- and subscale components in relevant thermo-vacuum environments. Specific analyses include: maximum mirror substrate size, first fundamental mode frequency (i.e., stiffness) and mass required to fabricate without quilting, survive launch, and achieve stable pointing and maximum thermal time constant.
\end{abstract}

